\title{
Closing up on dark sectors at colliders: From 14 to $100 \mathrm{TeV}$
}

\author{
Philip Harris, ${ }^{1, *}$ Valentin V. Khoze ${ }^{2, \dagger}$ Michael Spannowsky, ${ }^{2, \ddagger}$ and Ciaran Williams ${ }^{3, \$}$ \\ ${ }^{1}$ CERN, CH-1211 Geneva 23, Switzerland \\ ${ }^{2}$ Institute for Particle Physics Phenomenology, Department of Physics, Durham University, \\ Durham DH1 3LE, United Kingdom \\ ${ }^{3}$ Department of Physics, University at Buffalo The State University of New York, \\ Buffalo, New York 14260-1500, USA
}

(Received 26 October 2015; published 18 March 2016)

\begin{abstract}
We investigate the reach of the LHC Run 2 and that of a future circular hadron collider with up to $100 \mathrm{TeV}$ center of mass energy for the exploration of potential dark matter sectors. These dark sectors are conveniently and broadly described by simplified models. The simplified models we consider provide microscopic descriptions of interactions between the standard model partons and the dark sector particles mediated by the four basic types of ( $s$-channel) messenger fields: scalar, pseudoscalar, vector or axialvector. Our analysis extends and updates the previously available results for the LHC at 8 and $14 \mathrm{TeV}$ to $100 \mathrm{TeV}$ for models with all four messenger types. We revisit and improve the analysis at $14 \mathrm{TeV}$, by studying a variety of analysis techniques, concluding that the most discriminating variables correspond to the missing transverse energy and the azimuthal angle between jets in the final state. Going to $100 \mathrm{TeV}$, the limits on simplified models of dark matter are enhanced significantly, in particular for heavier mediators and dark sector particles, for which the available phase space at the LHC is restricted. The possibility of a $100 \mathrm{TeV}$ collider provides an unprecedented coverage of the dark sector basic parameters and a unique opportunity to pin down the particle nature of dark matter and its interactions with the standard model.
\end{abstract}

DOI: 10.1103/PhysRevD.93.054030

\section{INTRODUCTION}

The data collected by the Planck mission [1] confirms that, based on the standard model of cosmology, dark matter constitutes nearly $85 \%$ of the total matter content in the universe. With a natural assumption that all matter in the universe, dark and visible, is fundamental, dark matter should be described by a microscopic particle theory. ${ }^{1}$ Hence the quest to establish the identity of dark matter, and its fundamental interactions, amounts to one of the most important goals in particle physics.

The observational evidence for dark matter (DM) was established from gravitational effects on visible matter. However, the standard model (SM) of particle physics does not contain any viable DM candidates. In this way dark matter provides us with arguably the strongest experimental evidence for the existence of physics beyond the standard model. The observation of nongravitational interactions of DM with visible matter could be crucial in discovering extensions of known fundamental theories. Since the

\footnotetext{
*philip.coleman.harris@cern.ch

valya.khoze@durham.ac.uk

*michael.spannowsky@durham.ac.uk

§ciaranwi@buffalo.edu

${ }^{1}$ for a review see e.g. [2].
}

Published by the American Physical Society under the terms of the Creative Commons Attribution 3.0 License. Further distribution of this work must maintain attribution to the author(s) and the published article's title, journal citation, and DOI. experimental evidence for the existence of DM is currently only gravitational, at present we have no definitive microscopic understanding of dark matter. For these reasons the search for DM has escalated in recent years with direct detection, indirect detection, and most recently high energy collider searches combining to restrict the range of parameter space for dark matter models.

Presently the frontier of high-energy physics corresponds to the newly upgraded LHC, which is currently operating at $\sqrt{s}=13 \mathrm{TeV}$, with a planned increase to $\sqrt{s}=14 \mathrm{TeV}$ in the near future. However, it is certainly plausible that the scale of new physics is out of range of the LHC's reach. Broadly speaking, analyses of Run 1 data are able to constrain new physics approximately up to $2 \mathrm{TeV}$. The higher operating energy at Run 2 will certainly increase these bounds, but not by more than an order of magnitude. Therefore if new physics arises first at scales greater than those probed by the LHC, it may not be possible to infer its existence with the current generation of machines. For this reason, the community is beginning to consider the potential for the successor to the LHC [3]. The next machine will have to significantly extend the reach of the LHC, and as such, requires a large increase in operating energy. Current proposals set $100 \mathrm{TeV}$ as the target center of mass energy. Since a hadron machine of this energy mandates a circular design the putative machine is usually referred to as the Future Circular Collider (FCC). Such a machine would be a formidable tool in the quest to discover new physics. 
The main motivation of this paper is to investigate the reach of the FCC with a $100 \mathrm{TeV}$ center of mass energy for dark matter searches and ideally potential discoveries. In parallel with our earlier work [4] which analyzed the limits of the LHC at $8 \mathrm{TeV}$ and its DM discovery potential at $14 \mathrm{TeV}$, we aim for a general model-independent characterization of dark sectors with as few free parameters as possible, but without resorting to an effective field theory description. To achieve this we employ the simplified model approach which provides a microscopic QFT description of a minimal set of interactions between the standard model partons and the dark sector particles. These interactions are mediated by a complete set of four basic types of messenger fields, i.e. the scalar, pseudoscalar, vector and axial-vector. One naturally expects that the FCC should perform significantly better than the LHC for heavy mediators and heavy dark matter particles, the primary aim of this work is to seek to quantify this improvement. Our work is another step in the emerging program of DM studies at future colliders in the $100 \mathrm{TeV}$ range, [5-9]. Related studies using simplified models for constraining dark sectors at the LHC include Refs. [10-19], and we also refer the reader to the recent summaries [20,21] and references therein.

In DM searches at hadron colliders, the putative dark particles are pair-produced in collisions of the visible sector particles - the standard model quarks and gluons. In the setup we study here, there are no direct interactions between the SM sector and the dark matter particles. Instead these interactions are mediated by an intermediate degree of freedom - the mediator field. In general, one can expect four types of mediators, scalar $S$, pseudoscalar $P$, vector $Z^{\prime}$ or axial-vector $Z^{\prime \prime}$. The corresponding four classes of simplified models describing elementary interactions of these four mediators with the SM quarks and with the dark sector fermions $\chi$ are

$$
\mathcal{L}_{\text {scalar }} \supset-\frac{1}{2} m_{\mathrm{MED}}^{2} S^{2}-g_{\mathrm{DM}} S \bar{\chi} \chi-\sum_{q} g_{\mathrm{SM}}^{q} S \bar{q} q-m_{\mathrm{DM}} \bar{\chi} \chi
$$

$$
\begin{aligned}
\mathcal{L}_{\text {pseudo-scalar }} \supset & -\frac{1}{2} m_{\mathrm{MED}}^{2} P^{2}-i g_{\mathrm{DM}} P \bar{\chi} \gamma^{5} \chi \\
& -\sum_{q} i g_{\mathrm{SM}}^{q} P \bar{q} \gamma^{5} q-m_{\mathrm{DM}} \bar{\chi} \chi
\end{aligned}
$$

$$
\begin{aligned}
\mathcal{L}_{\text {vector }} \supset & \frac{1}{2} m_{\mathrm{MED}}^{2} Z_{\mu}^{\prime} Z^{\prime \mu}-g_{\mathrm{DM}} Z_{\mu}^{\prime} \bar{\chi} \gamma^{\mu} \chi \\
& -\sum_{q} g_{\mathrm{SM}}^{q} Z_{\mu}^{\prime} \bar{q} \gamma^{\mu} q-m_{\mathrm{DM}} \bar{\chi} \chi,
\end{aligned}
$$

$$
\begin{aligned}
\mathcal{L}_{\text {axial }} \supset & \frac{1}{2} m_{\mathrm{MED}}^{2} Z_{\mu}^{\prime \prime} Z^{\prime \prime \mu}-g_{\mathrm{DM}} Z_{\mu}^{\prime \prime} \bar{\chi} \gamma^{\mu} \gamma^{5} \chi \\
& -\sum_{q} g_{\mathrm{SM}}^{q} Z_{\mu}^{\prime \prime} \bar{q} \gamma^{\mu} \gamma^{5} q-m_{\mathrm{DM}} \bar{\chi} \chi
\end{aligned}
$$

The coupling constant $g_{\mathrm{DM}}$ characterizes the interactions of the messengers with the dark sector particles, which for simplicity we take to be Dirac fermions $\chi, \bar{\chi}$, the case of scalar DM particles is a straightforward extension of these results.

The coupling constants linking the messengers to the SM quarks are collectively described by $g_{\mathrm{SM}}^{q}$,

scalar \& pseudoscalar messengers: $g_{\mathrm{SM}}^{q} \equiv g_{q} y_{q}=g_{q} \frac{m_{q}}{v}$,

vector \& axial-vector messengers: $g_{\mathrm{SM}}^{q}=g_{\mathrm{SM}}$.

For scalar and pseudoscalar messengers the couplings to quarks are taken to be proportional to the corresponding Higgs Yukawa couplings, $y_{q}$ as in models with minimal flavour violation [22], and we keep the scaling $g_{q}$ flavoruniversal for all quarks. For axial and vector mediators $g_{\mathrm{SM}}$ is a gauge coupling in the dark sector which we also take to be flavor universal. The coupling parameters which we can vary are thus $g_{\mathrm{DM}}$ plus either $g_{q}$ or $g_{\mathrm{SM}}$, the latter choice depending on the messengers. ${ }^{2}$

In general, the simplified model description of the dark sector is characterized by five parameters: the mediator mass $m_{\mathrm{MED}}$, the mediator width $\Gamma_{\mathrm{MED}}$, the dark particle mass $m_{\mathrm{DM}}$, and the mediator-SM and the mediator-dark sector couplings, $g_{\mathrm{SM}}, g_{\mathrm{DM}}$. Out of these, the mediator width $\Gamma_{\mathrm{MED}}$, does not appear explicitly in the simplified model Lagrangians (1)-(4) and should be specified separately. $\Gamma_{\text {MED }}$ accounts for the allowed decay modes of a given mediator particle into other particles from the visible and the dark sector. In a complete theory, $\Gamma_{\mathrm{MED}}$ can be computed from its Lagrangian, but in a simplified model we can instead determine only the so-called minimal width $\Gamma_{\mathrm{MED} \text {,min }}$, i.e. the mediator width computed using the mediator interactions with the SM quarks and the $\bar{\chi}, \chi$ DM particles defined in Eqs. (1)-(4). Importantly $\Gamma_{\text {MED,min }}$ does not take into account the possibility of the mediator to decay into e.g. other particles of the dark sector, beyond $\bar{\chi}, \chi$, which would increase the value of $\Gamma_{\mathrm{DM}}$. In Ref. [4] we have investigated the role of $\Gamma_{\text {MED }}$ as an independent parameter in the simplified models characterization of dark sectors by using a simple grid for $\Gamma_{\mathrm{DM}}=\{1,2,5,10\} \times \Gamma_{\text {MED,min }}$.

Here we will not repeat this analysis, referring to [4] for a more general discussion of $\Gamma_{\mathrm{MED}}$; we will instead adopt a reduced simplified description where the width is set to its minimal computed value $\Gamma_{\mathrm{MED} \text {,min }}$ which amounts to larger signal cross-sections (we will also check that $\Gamma_{\mathrm{MED} \text {,min }}<m_{\mathrm{MED}} / 2$ ). For our simplified models we have

\footnotetext{
${ }^{2}$ In Ref. [4] we have parametrized $g_{\mathrm{DM}}$ for (pseudo)scalar messengers as $g_{\mathrm{DM}}=g_{\chi} m_{\mathrm{DM}} / v$ to look symmetric with respect to (5), and have treated $g_{\chi}$ as a free parameter. Here we do not impose this requirement and leave $g_{\mathrm{DM}}$ as the free parameter.
} 


$$
\Gamma_{\text {MED,min }}=\Gamma_{\chi \bar{\chi}}+\sum_{i=1}^{N_{f}} N_{c} \Gamma_{q_{i} \bar{q}_{i}}
$$

where $\Gamma_{\chi \bar{\chi}}$ is the mediator decay rate into two DM fermions, and the sum is over the SM quark flavors. Depending on the mediator mass, decays to top quarks may or may not be open i.e. $m_{\mathrm{MED}}$ should be $>2 m_{t}$ for an open decay. The partial decay widths of vector, axial-vector, scalar and pseudoscalar mediators into fermions are given by,

$$
\begin{gathered}
\Gamma_{f \bar{f}}^{V}=\frac{g_{f}^{2}\left(m_{\mathrm{MED}}^{2}+2 m_{f}^{2}\right)}{12 \pi m_{\mathrm{MED}}} \sqrt{1-\frac{4 m_{f}^{2}}{m_{\mathrm{MED}}^{2}}}, \\
\Gamma_{f \bar{f}}^{A}=\frac{g_{f}^{2}\left(m_{\mathrm{MED}}^{2}-4 m_{f}^{2}\right)}{12 \pi m_{\mathrm{MED}}} \sqrt{1-\frac{4 m_{f}^{2}}{m_{\mathrm{MED}}^{2}}} \\
\Gamma_{f \bar{f}}^{S}=\frac{g_{f}^{2}}{8 \pi} m_{\mathrm{MED}}\left(1-\frac{4 m_{f}^{2}}{m_{\mathrm{MED}}^{2}}\right)^{\frac{3}{2}}, \\
\Gamma_{f \bar{f}}^{P}=\frac{g_{f}^{2}}{8 \pi} m_{\mathrm{MED}}\left(1-\frac{4 m_{f}^{2}}{m_{\mathrm{MED}}^{2}}\right)^{\frac{1}{2}}
\end{gathered}
$$

where $m_{f}$ denotes masses of either SM quarks $q$ or DM fermions $\chi$ and the coupling constant $g_{f}$ denotes either $g_{\mathrm{SM}}$ or $g_{\mathrm{DM}}$.

In this paper we will focus on jets plus missing energy searches, generalizing our earlier results [4] from the 8-14 TeV to $100 \mathrm{TeV}$ colliders. The analysis in [4] was based on a monojet plus missing transverse energy (MET, or $E_{T}$ ) signature - a popular choice used in searches for new physics including supersymmetry, extra dimensions and dark matter at the Tevatron and the LHC [23-35]. Here we will update the experimental analysis techniques to take into account both the leading and trailing jets in the final state to present a more realistic idea of potential limits. Hence we will update our LHC 14 results [4] accordingly to provide a fair benchmark.

Depending on the choice for the mediator field different production mechanisms will contribute. For vectors and axial-vectors the dominant mechanism is the quark-antiquark annihilation at tree-level. For scalars and pseudoscalars on the other hand, the loop-level gluon fusion processes are more relevant. The representative Feynman diagrams for both channels are shown in Fig. 1. In comparing DM collider searches with direct and indirect detection experiments it is important to keep in mind that our collider processes and limits continue to be applicable for discovery of any dark sector particles escaping the detector. Hence dark particles produced at colliders do not have to be the cosmologically stable dark matter.

Finally we would like to comment on the possible origin and the UV consistency of the simplified models (1)-(4). The scalar and pseudoscalar messenger fields in our simplified models (1)-(2) are singlets under the standard model. How can this be reconciled with the fact that they
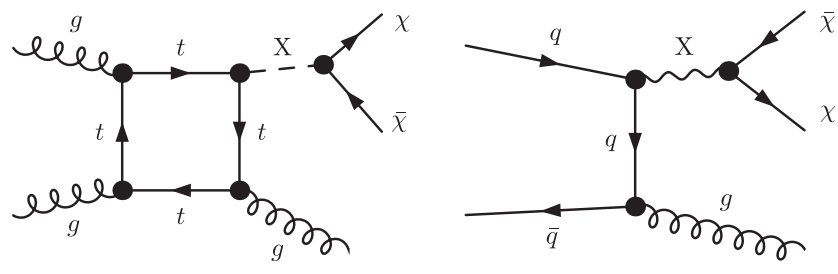

FIG. 1. Representative Feynman diagrams for gluon and quark induced monojet plus MET processes. The mediator X can be a scalar, pseudoscalar, vector or axial-vector particle. The gluon fusion process involves the heavy quark loop which we compute in the microscopic theory, while the quark-antiquark annihilation is a tree-level process at leading order.

are supposed to be Higgs-like, with the Higgs being an $S U(2)_{L}$ doublet? In fact, the simplified models (1)-(2) can arise from two types of the more fundamental theories. The simplest theories of the first type are the two-Higgs-doublet models [36]. In this case the mediators would originate from the second Higgs doublet. The other type of models giving rise to our simplified models are even simpler in the sense that scalar mediators (and the dark sector particles they are coupled to) can be genuinely neutral under the SM but mix with the neutral component of the Higgs. Following the Higgs discovery there is a renewed interest in the literature in Higgs portal models [37-40] where the scalar mediators are SM-singlets but the SM Higgs $h$ interacts with them via the interaction, $\lambda_{\mathrm{hp}}|H|^{2}|\Phi|^{2}$. When $\Phi$ and $H$ both develop a VEV, mass mixing occurs, and after transforming into the mass eigenstate basis, one finds two scalar resonances $h_{1}$ and $h_{2}$, both of which interact with the Standard Model and the dark sector, with the $h_{1}$ state identified with the SM Higgs and $h_{2}$ being the scalar mediator. These models provide a direct connection of the dark sector with Higgs physics and can link the origin of the electroweak and the DM scales [41-44]. The simplified dark sector models with vector and axial-vector mediators in Eqs. (3)-(4) can also be derived from appropriate firstprinciples theories. Since the mediators are spin-one particles, these UV models would necessarily require the mediators to be gauge fields and the DM to be charged under these gauge transformations. A classification of anomaly-free extensions of the Standard Model Abelian $U(1)^{\prime}$ factor was given in [45] and can be used for constructing an example of a consistent gauge-invariant vector and axial theories of the type (4).

This paper proceeds as follows, in Sec. II we discuss the theoretical and experimental setup we used for our analysis. The results of our analysis are presented in Sec. III, and our conclusions are presented in Sec. IV.

\section{THEORETICAL AND EXPERIMENTAL SETUP}

\section{A. Collider design and Monte Carlo generation}

At this moment, the exact nature of the FCC is unclear. This level of uncertainty includes fundamental issues, like the operating center of mass energy of the machine, and 
technical details including, for instance, the capabilities of future detectors. This makes robust predictions impossible at the present time, instead it is more interesting to study what is potentially feasible at future colliders, given a modest (and conservative) set of assumptions.

We begin by discussing the straightforward benefits associated with higher energy machines in relation to DM searches. Clearly with larger center of mass energies, heavier mediators can be produced on-shell, allowing for significant enhancements for heavy mediators compared to a $14 \mathrm{TeV}$ analysis. Second, there are effects due to the parton distribution functions (PDFs). A typical $14 \mathrm{TeV}$ DM analysis naturally focuses on the region of high missing transverse energy, which corresponds to larger values of the partonic center of mass energy $(\hat{s})$. This region of phase space is suppressed by the PDFs, and therefore additionally damps the differential cross section, beyond the simple phase space/matrix element falloff. However, at higher energies the same region of phase space now corresponds to much smaller $\hat{s} / s$ values, and as a result the PDF suppression is no longer as severe. Therefore, even for mass scales which are currently probed at the LHC, we expect a higher energy machine to be able to improve upon the bounds.

A major concern for the simulation of analyses at an FCC, is the modeling of the theoretical event generation. It is clear that a huge amount of work will be needed over the coming decades to ensure that the Monte Carlo simulations provide a reasonable description of signal and background processes [46]. We do not attempt this huge feat in this paper, but instead list the concerns regarding existing tools, and their potential impact.

First, there is the obvious issue regarding tuning and PDF fits, which have been undertaken at much lower energy than those accessible at FCC's. Of particular importance are the extraction of the gluon PDF's, which will dominate the initial state configurations. Second, there is the accuracy of the perturbative component of the simulations. This is particularly worrisome. At FCC energies emission of additional radiation will result in copious jet-production around the electroweak scale. This will require delicate handling with respect to matching and merging of parton shower and matrix element emissions. Since matching prescriptions typically require scales which separate emissions into the two categories, one can easily imagine existing tools for LHC physics are not optimal for future FCC predictions. An additional concern relates to the simulation of electroweak bosons, at $100 \mathrm{TeV}$, the mass of the $W, Z$ and $H$ bosons become small scales, and Sudakov logarithms associated with their emission from partons becomes relevant. The resolution of these issues will take many years of research and improvements to existing tools. However given the likely timescale of construction, and the rapid improvement in theoretical tools, none of the above issues should be regarded as significantly likely to negatively affect the physics program at the FCC. They should certainly be kept in mind and the resulting theoretical predictions used in this paper should be interpreted as having a large uncertainty.

In order to attempt to simulate events at the FCC it is therefore critical to include high energy jet radiation in the best manner possible. This can be achieved by ensuring that matched samples are used. Backgrounds are generated at next to leading order for $0,1,2$ jets merged using MadGraph-aMC@NLO [47], with the exception of $W+$ jets which does not have the second jet merged. Diboson and single top processes are all included. For the signal we use MadGraph for the vector/aAxial simplified models and a combination of MCFM [48,49] and VBFNLO [50] for the production of scalar/pseudoscalar mediators in association with one and two-jets. The output LHE events are then merged using the CKKW-L interface of Pythia 8 [51]. NNPDF3.0 [52] PDF's are used for the generation of all Monte-Carlo samples. In order to generate the samples consistently the mediator is assumed to be on-shell for the 1 and 2 jet merged sample. The one-jet sample is generated with the full width and thus includes off-shell effects. Since the putative mediators scrutinized at the FCC will include heavier masses, off-shell effects will indeed play an important role in the simulation. However, given the large center of mass energy, including at least the two-jet matrix element (and ideally in the future 3-jet and beyond) is likely to be a more important requirement than including off-shell effects. ${ }^{3}$ In the following section we therefore present results both for the one-jet sample (with the full off-shell effects) and the two-jet merged sample (which includes hard matrix element jets, but requires on-shell mediators). We note that this is not an issue for the vector/axial mediators. In all cases we calculate the minimum width which corresponds to all open fermionic decay channels [see Eqs. (7)-(9) for definitions].

In addition to the theoretical issues discussed above, we have limited knowledge of future detectors. However, here a conservative assumption can easily be made. We simply assume in this paper, that the detector at the FCC is a copy of the CMS detector. One extension to this detector is added, the lepton acceptance for this upgraded detector is assumed to extend up to $|\eta|<4.0$ and $|\eta|<5.5$ for the $14 \mathrm{TeV}$ and $100 \mathrm{TeV}$ detector respectively. This design is consistent with the plans for the upgraded CMS and ATLAS detectors $[55,56]$. The choice of detector allows us to utilize the extensive studies of the detector performance. In reality we can expect improvements in technology which can significantly improve on existing detectors.

To emulate the effects of a detector, the MET is smeared as a function of the mediator $p_{\mathrm{T}}$ [57]. The jets are smeared

\footnotetext{
${ }^{3}$ We note that very recently Refs. [53,54] presented an implementation of the signal model which can incorporate one- and two-jet merged samples and off-shell effects.
} 

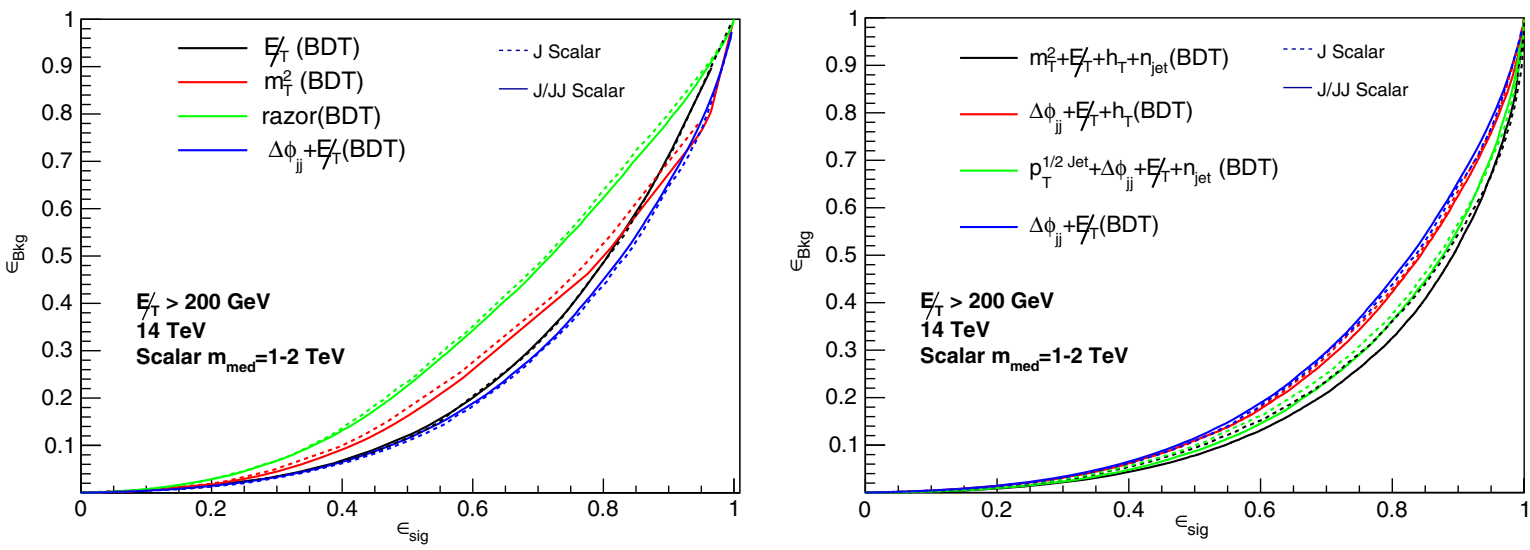

FIG. 2. Reconstruction efficiency for signal $\epsilon_{\text {sig }}$ and background $\epsilon_{\mathrm{bkg}}$ as functions of individual kinematic variables at $14 \mathrm{TeV}$ with $1 \mathrm{ab}^{-1}$ luminosity for scalar mediator models using single variables as well as the combinations of variables, as indicated. The dashed lines depict single jet signal MC, while the solid lines correspond to merged samples with up to two jets.

as a function of $\eta$ and $p_{\mathrm{T}}$ [58]. Effects from pileup are taken to account, such that the respected observed resolution follows closely the expected conditions for high luminosity running at the LHC.

\section{B. Kinematic distributions for signal and background}

In light of using multileg Monte Carlos and recent developments in shape based analysis for dark matter projections [59], the analysis selection was reoptimized from a previous cut and count analysis by considering a shape based analysis in both single and pairwise combinations of variables that take into account both the leading and trailing jets. To find sensitive variables to separate signal from backgrounds we focused on $E_{T}, m_{T}^{2}$, the razor variables, the scalar sum of all jets above $30 \mathrm{GeV}\left(h_{T}\right)$, and the angle between the two leading jets $\Delta \phi_{j j}$ [59-61]. Figure 2, shows the performance of these variables when compared with an amalgam of signal Monte Carlos. For these signal models, a dark matter mass of $50 \mathrm{GeV}$ is taken (ensuring on-shell decays for both). The range of samples consists of those with different mediator masses ranging from $1 \mathrm{TeV}$ to $2 \mathrm{TeV}$ in $100 \mathrm{GeV}$ intervals. The samples are combined by cross-section weights. We recall that the 1 jet Monte Carlo is generated with full on and off shell width effects, whereas the 1-jet/2-jet merged sample is generated explicitly on-shell. When considering the single variable performance, we find $E_{T}$ is the most discriminating variable. As a result, combining these variables into pairwise combinations using a boosted decision tree we find that the most sensitive combination is $E_{T}$ and $\Delta \phi_{j j}$ between the two leading jets. This can be readily seen in the left plot in Fig. 2 where the $\Delta \phi_{j j}+E_{T}$ combination is shown as the solid line in blue. In the lower-left quadrant of the plot the reconstruction efficiency of the signal $\epsilon_{\text {sig }}$ significantly exceeds that of the background, for example for $\epsilon_{\mathrm{sig}} \simeq 0.5$ we have $\epsilon_{\mathrm{bkg}} \simeq 0.1$ on the blue contour in
Fig. 2. A comparison with higher dimensional sets of variables in the right panel shows even further improvement, particularly when the second jet merged MC is used. However, for this further optimization to manifest itself in the analysis requires precise knowledge of the kinematic discrimination of $E_{T}$ in the far tails of the distribution. Additionally we note that the larger differences between the one-jet and two-jet signal MC, can be indicative of greater sensitivity to theoretical modeling. Thus, we do not extend these studies beyond their current projections. Our final extractions are then performed by fitting the $E_{T}$ distribution in two bins of $\Delta \phi_{j j}\left(\Delta \phi_{j j}<1.1\right.$ and $\left.\Delta \phi_{j j}>1.1\right)$. The binning is optimized such that the full spectrum is covered with MC samples. Additionally a selection of $\min \left(\Delta \phi_{E_{T}, j_{i}}\right) \geq 0.5$, where $i$ runs over all jets is applied to minimize the impact of QCD multijet events where one or several of the jets are mismeasured. As a result of this selection being applied, this background is neglected throughout the course of these studies. Further improvements could be obtained by using finer bins for the tails of the distribution. ${ }^{4}$

The signal extraction is performed with a full shape analysis in which the shape for the dominant backgrounds are taken from control regions. For the $Z \rightarrow \nu \bar{\nu}$ background, the $Z \rightarrow \mu^{+} \mu^{-}$control region is used as a background. For the $W \rightarrow \ell \nu$, top and diboson backgrounds, we use the single lepton control region. For each of these control regions the full statistical uncertainty on the shape is propagated per bin on each of the backgrounds with an additional one percent uncertainty uncorrelated per bin to account for additional modeling uncertainties. For all but the tail bins of the shape uncertainties on the $E_{T}$ spectrum

\footnotetext{
${ }^{4}$ While we will not include this approach in our analysis we point out that this could be a viable way to optimize the sensitivity of the search.
} 
are roughly $1 \%$ with the dominant uncertainty resulting from the additional one percent modeling uncertainty. Finally, the signal is profiled using the standard limit extraction $\left(\mathrm{CL}_{s}\right)$ [62,63]. Additional nuisances are placed on the background normalization for lepton efficiencies and luminosity. These are constrained to very small values due to the large data set in the signal region and do not affect the limit sensitivity.

Kinematic distributions for our simplified models of dark sectors alongside the main SM backgrounds are shown in Figs. 3, 4 and 5. The distributions are shown as functions of four kinematic variables, $p_{T}$ of the leading jet, $p_{T}$ of the trailing jet, the azimuthal angle between the jets $\cos \left(\Delta \phi_{j j}\right)$, and missing energy $E_{T}$.
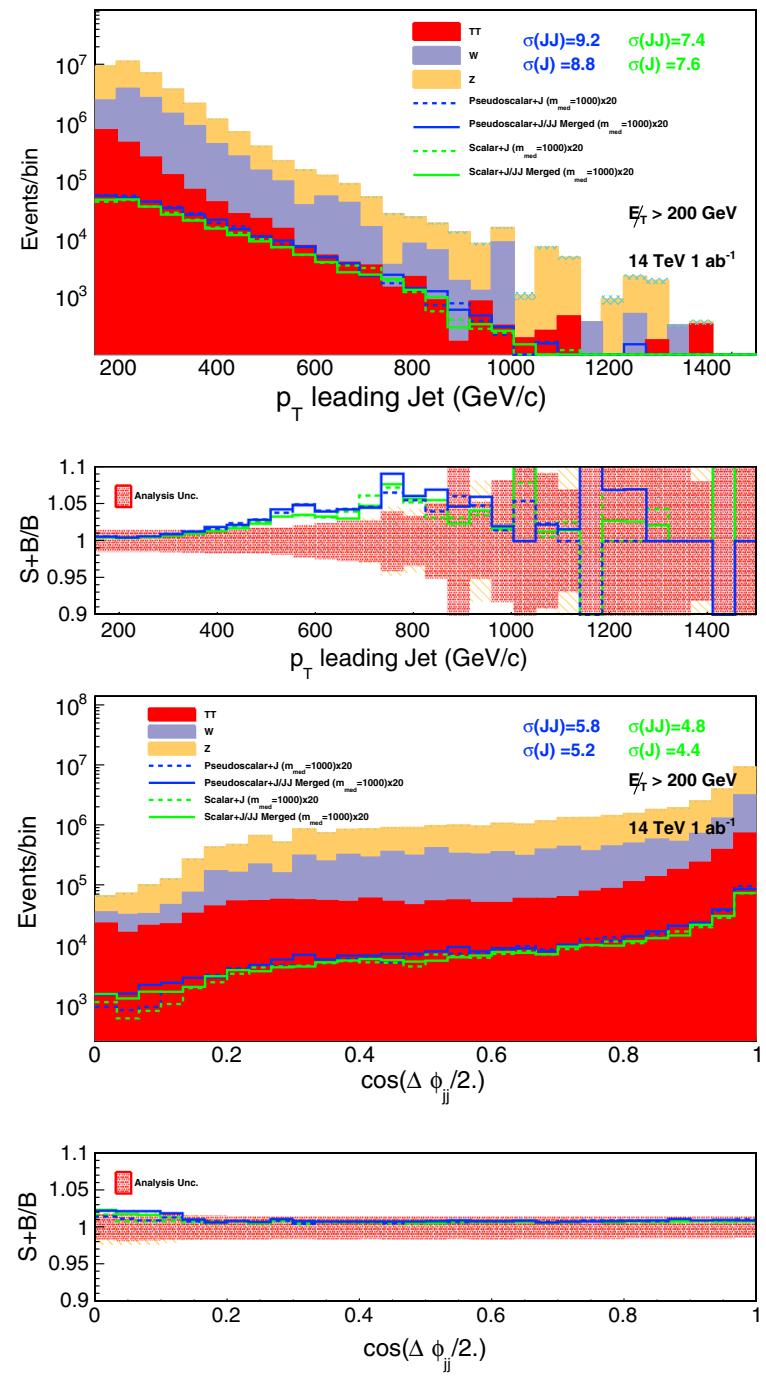

In Figs. 3 and 4 we show shapes of kinematic distributions corresponding to the (pseudo)scalar and (axial-)vector case respectively, with the signals and backgrounds computed at $14 \mathrm{TeV}$ center-of-mass energy and assuming $1 \mathrm{ab}^{-1}$ integrated luminosity. The only event selection cuts imposed for the distributions in Figs. 3-5 are $E_{T} \geq$ $200 \mathrm{GeV}$ and $\min \left(\Delta \phi_{E_{T}, j_{i}}\right) \geq 0.5$, where $i$ runs over all jets in each event.

We find that for the event selection cuts imposed, the distributions for samples using multijet merging are similar to the unmerged samples. In the gluon-induced scalar and pseudoscalar cases the unmerged samples result in a harder $p_{T}$ distribution, particularly for the trailing jet. On the other hand, the $\cos \left(\Delta \phi_{j j}\right)$ distribution changes more
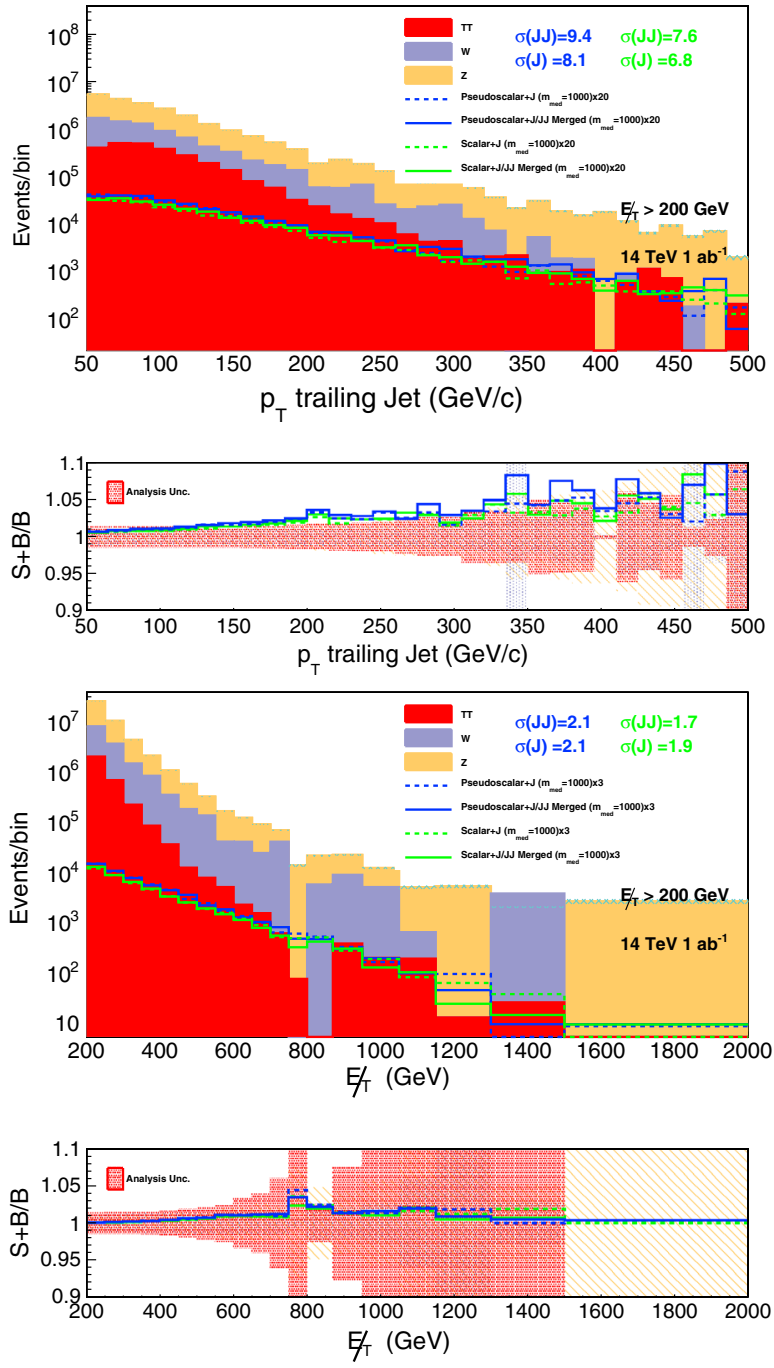

FIG. 3. Kinematic distributions for signal scalar and mediator models and the SM backgrounds at $14 \mathrm{TeV}^{\mathrm{assuming}} 1 \mathrm{ab}^{-1}$ of integrated luminosity. We show four kinematic variables: $p_{T}$ of the leading jet, $p_{T}$ of the trailing jet, the azimuthal angle between the jets $\cos \left(\Delta \phi_{j j}\right)$, and the missing energy $E_{T}$. Ratios of $(S+B) / B$ are shown for each observable. The red bands indicate the uncertainties on the background distributions. The accordingly color-coded numbers for $\operatorname{sig}(\mathrm{JJ})$ and $\operatorname{sig}(\mathrm{J})$ give the statistical significance to disfavor the presence of the signal using the $\mathrm{CL}_{s}$ method. 

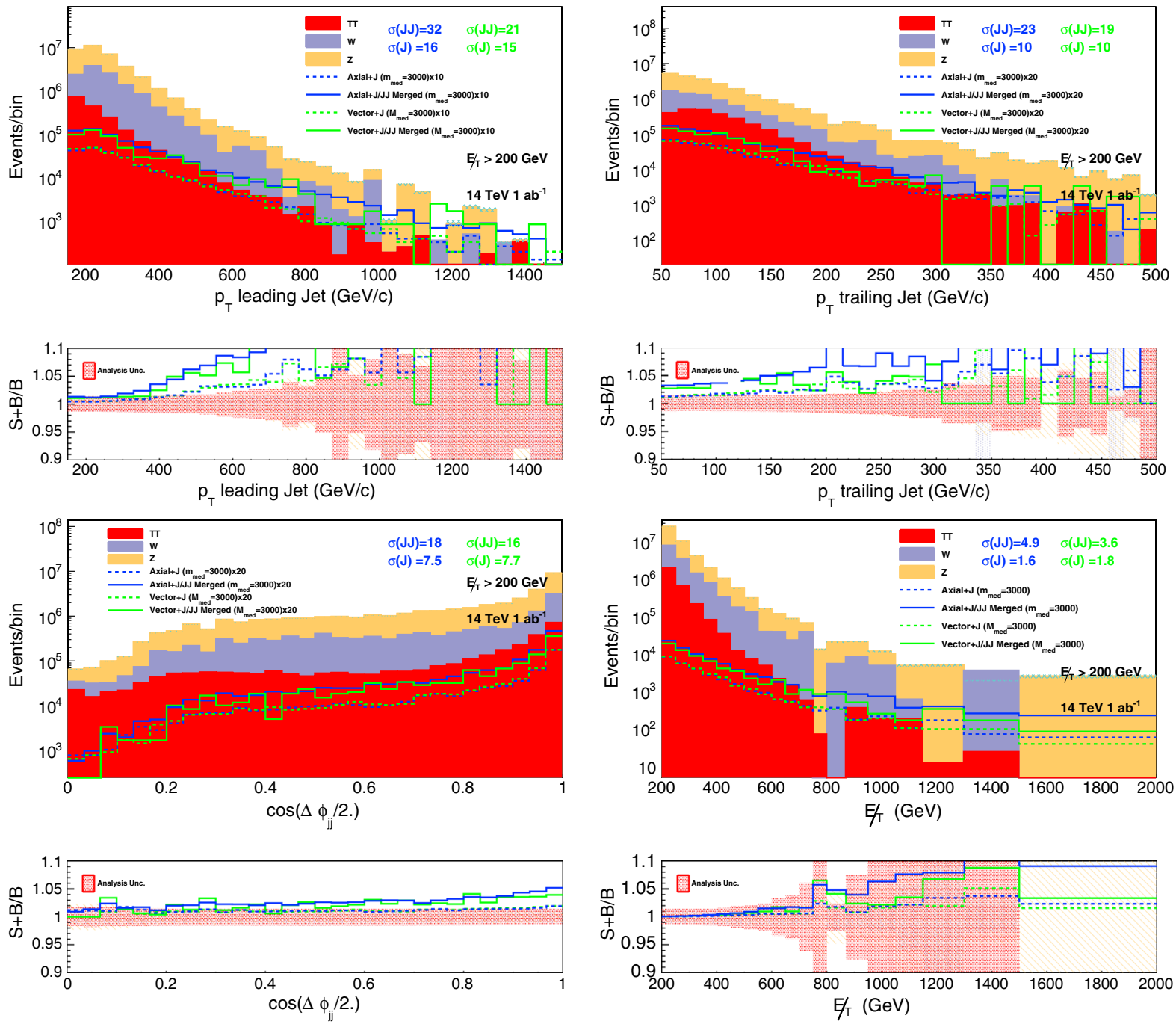

FIG. 4. Kinematic distributions for signal vector and axial-vector models and the SM backgrounds at $14 \mathrm{TeV}^{\mathrm{assuming}} 1 \mathrm{ab}^{-1}$ of integrated luminosity. We show four kinematic variables: $p_{T}$ of the leading jet, $p_{T}$ of the trailing jet, the azimuthal angle between the jets $\cos \left(\Delta \phi_{j j}\right)$, and the missing energy $E_{T}$. Ratios of $(S+B) / B$ are shown for each observable. The red bands indicate the uncertainties on the background distributions. The accordingly color-coded numbers for $\operatorname{sig}(\mathrm{JJ})$ and $\operatorname{sig}(\mathrm{J})$ give the statistical significance to disfavour the presence of the signal using the $\mathrm{CL}_{s}$ method.

rapidly when including multijet merging. In Fig. 5 we show the kinematic distributions for the two most discriminating variables, which, as was pointed out above, are the missing transverse energy $E_{T}$ and the azimuthal angle between the jets $\Delta \phi_{j j}$ at $\sqrt{s}=100 \mathrm{TeV}$. The two plots on the left show the vector and axial-vector case while the two plots on the right show the scalar and pseudoscalar signals.

\section{Direct and indirect detection limits}

In Sec. III we will compare collider limits with limits from direct and indirect detection experiments. For convenience we recall the formulas for direct and indirect detection limits, in terms of the simplified models of Eq. (1)-(4).
We use

$$
\sigma_{\chi \mathrm{p}}^{V}=\frac{9}{\pi} \frac{g_{\mathrm{DM}}^{2} g_{\mathrm{SM}}^{2} \rho^{2}}{m_{\mathrm{MED}}^{4}}
$$

and

$$
\sigma_{\chi \mathrm{p}}^{A}=\frac{3}{\pi} \frac{g_{\mathrm{DM}}^{2} g_{\mathrm{SM}}^{2} a^{2} \rho^{2}}{m_{\mathrm{MED}}^{4}},
$$

with $a \simeq 0.43 \quad[64,65]$ and the reduced mass $\rho=m_{\mathrm{DM}} m_{p} /\left(m_{\mathrm{DM}}+m_{p}\right)$, for the cross section of a dark matter particle scattering spin-independently (vector mediator) or spin-dependently (axial-vector mediator) from a proton. 

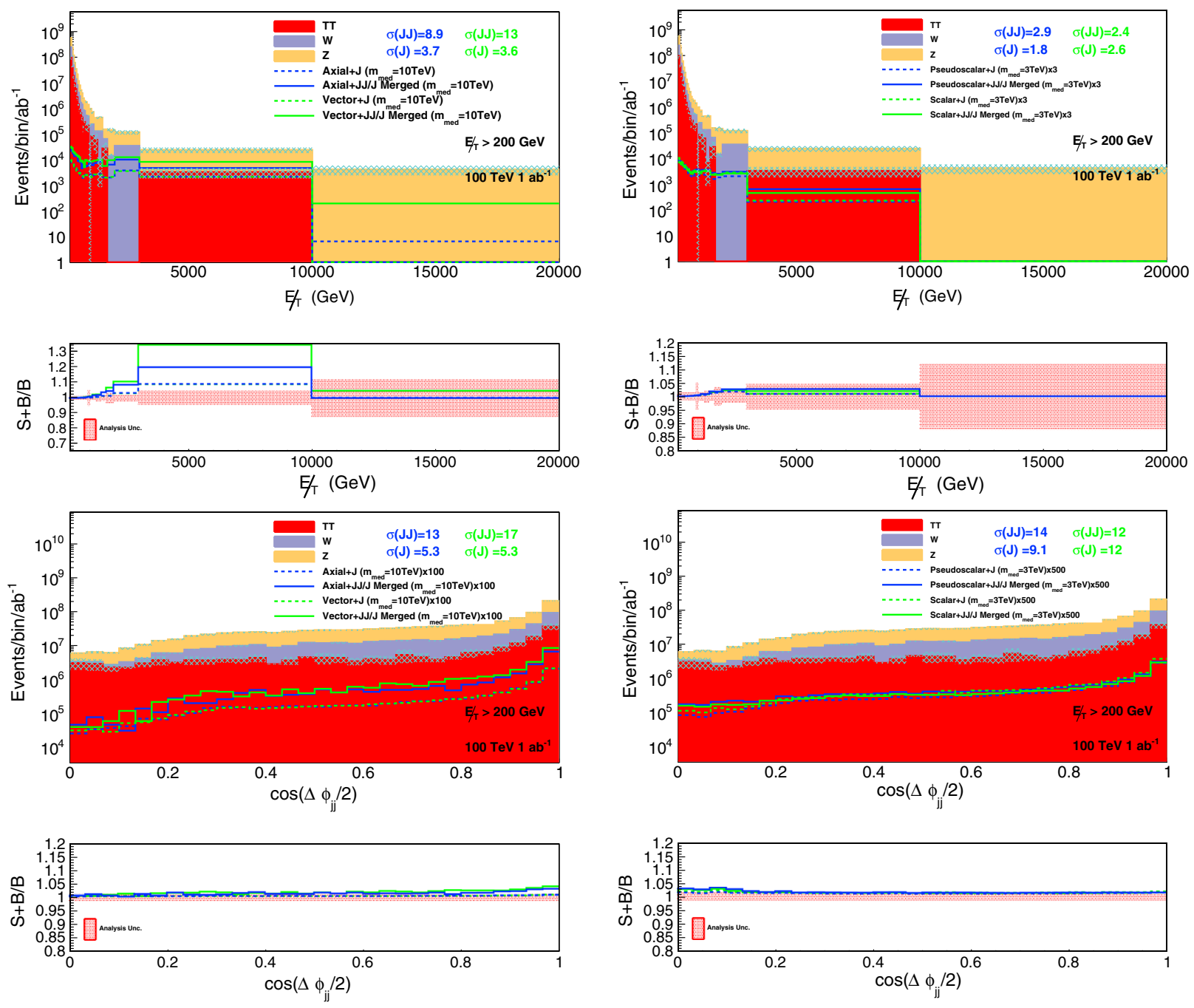

FIG. 5. Kinematic distributions for the two most discriminating variables $E_{T}$ and $\cos \left(\Delta \phi_{j j}\right)$ at $\sqrt{s}=100 \mathrm{TeV}$.

The cross section for a dark matter particle scattering from a nuclei via a scalar mediator of Eq. (1) is given by [66-68]

$$
\begin{aligned}
\sigma_{\chi \mathrm{p}}^{S}= & \frac{\rho^{2}}{\pi} \mid \sum_{q=u, d, s} f_{q}^{p} \frac{m_{p}}{m_{q}}\left(\frac{g_{\mathrm{DM}} g_{q} y_{q}}{m_{\mathrm{MED}}^{2}}\right) \\
& +\left.\frac{2}{27} f_{\mathrm{TG}} \sum_{q=c, b, t} \frac{m_{p}}{m_{q}}\left(\frac{g_{\mathrm{DM}} g_{q} y_{q}}{m_{\mathrm{MED}}^{2}}\right)\right|^{2},
\end{aligned}
$$

where $f_{u}^{p}=0.019, f_{d}^{p}=0.045, f_{s}^{p}=0.043$ and $f_{\mathrm{TG}} \simeq$ $1-\sum_{q=u, d, s} f_{q}^{n}[69,70]$ and $m_{p}$ is the proton mass.

When comparing the expected sensitivity for the LHC and FCC for DM searches to those of direct detection (DD) it is interesting to compare to expected impact of the neutrino wall $[12,71]$. The neutrino wall is a result of the background of cosmic neutrinos. We take their interaction cross section to be indicative for the ultimate reach of DD experiments. Therefore in the following section we will present the equivalent limit which may be obtained from
DD experiments with the currently hypothesized values of the neutrino wall $[12,71]$. For a pseudoscalar mediator, taking existing limits into account [72,73], indirect detection experiments can result in stronger limits than direct detection experiments [74,75]. For the simplified model of Eq. (2), using the velocity-averaged DM annihilation cross section into $\bar{b} b$,

$$
\langle\sigma v\rangle_{\bar{b} b}^{P}=\frac{N_{C}}{2 \pi} \frac{\left(y_{b} g_{b}\right)^{2} g_{\mathrm{DM}}^{2} m_{\mathrm{DM}}^{2}}{\left(m_{\mathrm{MED}}^{2}-4 m_{\mathrm{DM}}^{2}\right)^{2}+m_{\mathrm{MED}}^{2} \Gamma_{\mathrm{MED}}^{2}} \sqrt{1-\frac{m_{b}^{2}}{m_{\mathrm{DM}}^{2}}},
$$

which allows us to derive a limit on the parameters in the $\bar{b} b$ channel [72].

\section{RESULTS}

Results are obtained scanning over a spectrum of signal models at $14 \mathrm{TeV}$ and $100 \mathrm{TeV}$. A predicted luminosity of 
$1 \mathrm{ab}^{-1}$ is used for both analyses, so the sensitivity can be compared directly. We note that this amount of integrated luminosity is a rather modest amount compared to what is likely to be collected at a future collider, whereas for the LHC it corresponds to a significant fraction of the data set that will be obtained. As a result the results we present here should be interpreted as those which can be obtained over the lifetime of the LHC, and for a shorter run with the FCC.

We begin by studying constraints on total cross sections which can be obtained using our analysis. Figure 6 presents the total cross section which the analysis excludes for each of the four mediator types defined in Eqs. (1)-(4). We define our cross sections by setting $g_{\mathrm{DM}}=g_{\mathrm{SM}}=1$ and select the mediator mass as indicated in the legend of each figure respectively. As an illustrative example we have chosen a relatively small characteristic value of $100 \mathrm{GeV}$,
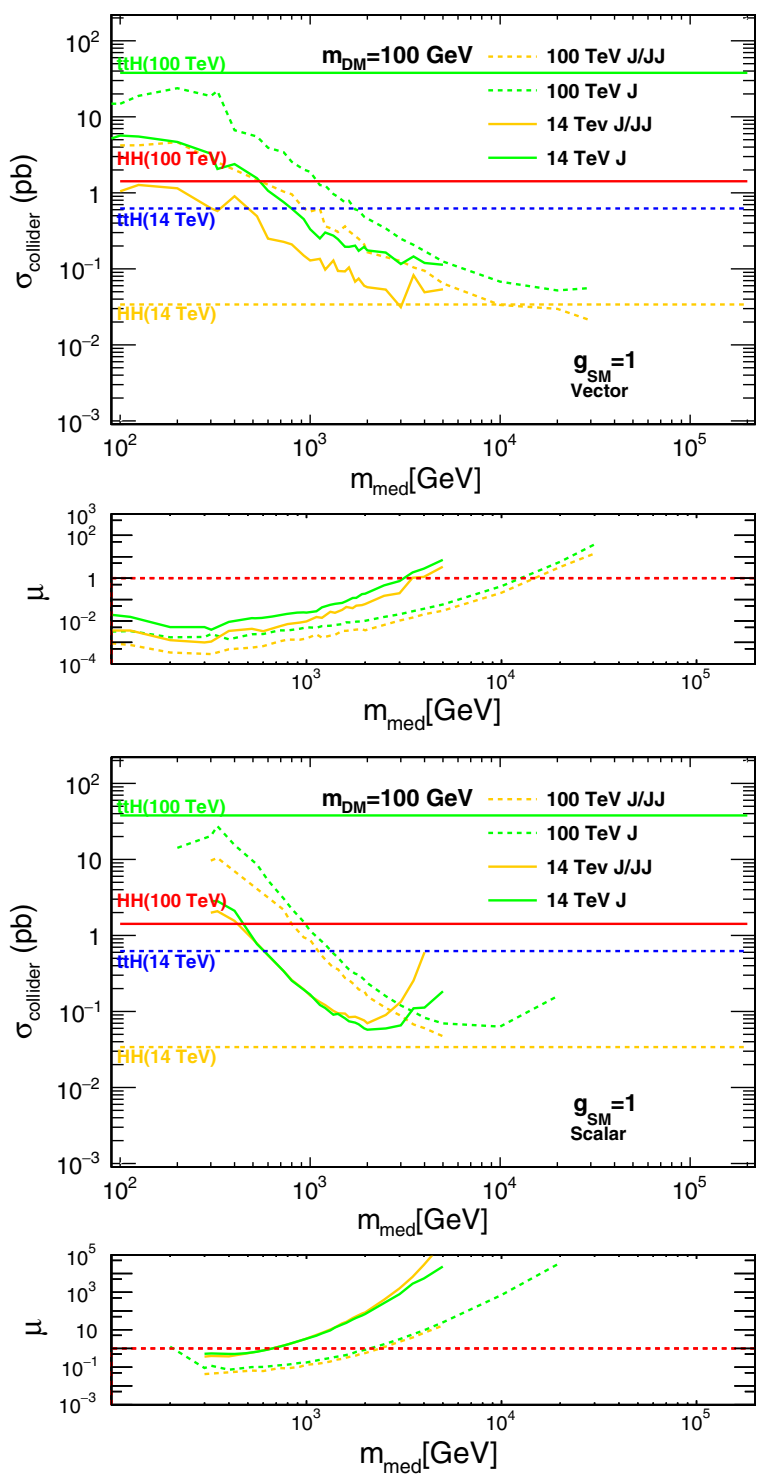

although the results obtained for other kinematically accessible values of dark matter mass were found to be similar. The kinematics of the process are then completely specified once the couplings $g_{\mathrm{DM}}$ and $g_{\mathrm{SM}}$ are set, since this fixes the minimal width of the mediator [4]. The excluded cross section is then related to the predicted cross section as follows,

$$
\sigma=\mu \quad \sigma\left(g_{\mathrm{DM}}=1, g_{\mathrm{SM}}=1, m_{\mathrm{MED}}\right)
$$

With the kinematics of the model fixed we set a limit on $\mu$ defined above using the $\mathrm{CL}_{s}$-method, again assuming $1 \mathrm{ab}^{-1}$ of data. Values with $\mu<1$ indicate the excluded couplings and width are smaller than the tested model, and the point is then excluded.
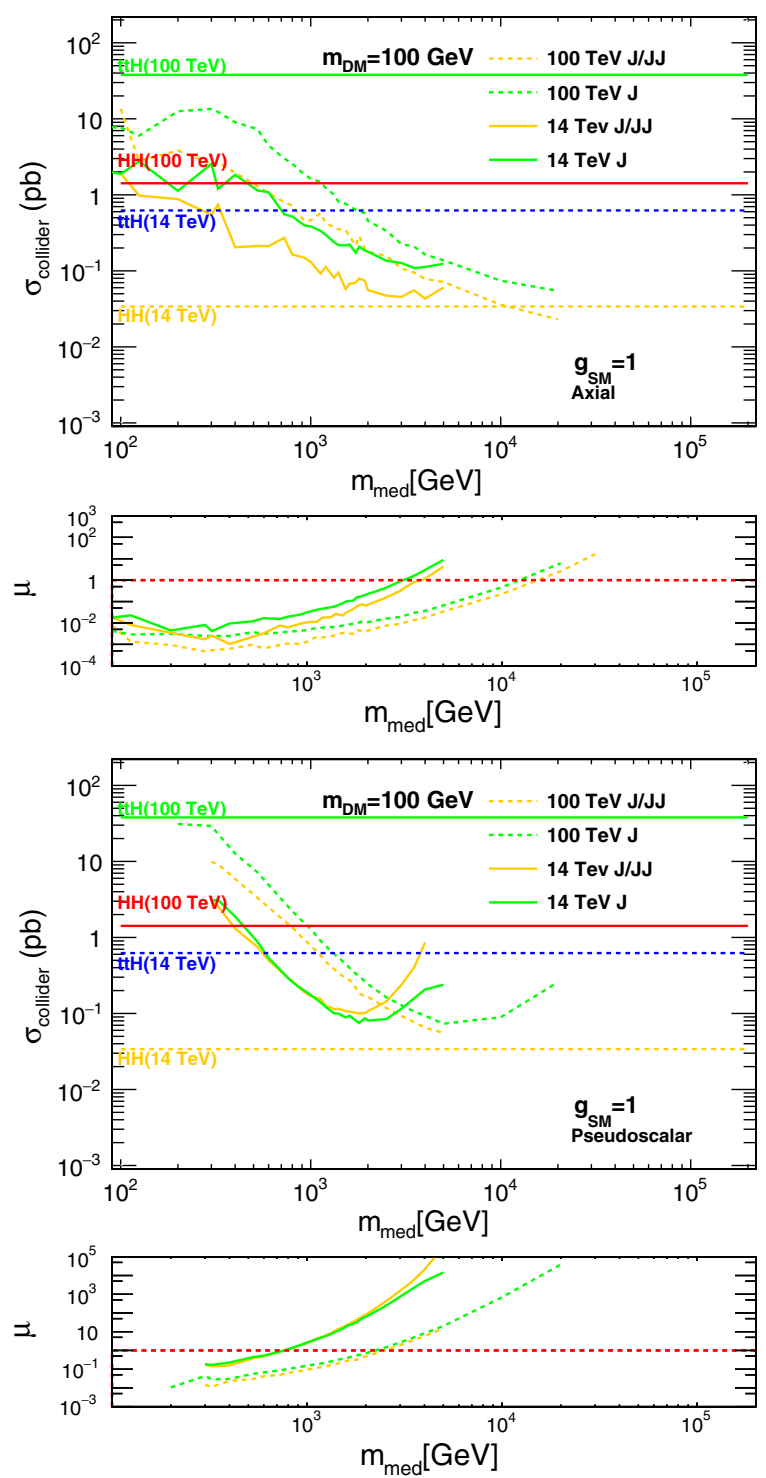

FIG. 6. Cross section exclusion limits as a function of mediator mass for a fixed dark matter mass at a given coupling. We show results for vector (upper left panel), axial-vector (upper right panel), scalar (lower left panel) and pseudoscalar (lower right panel). 

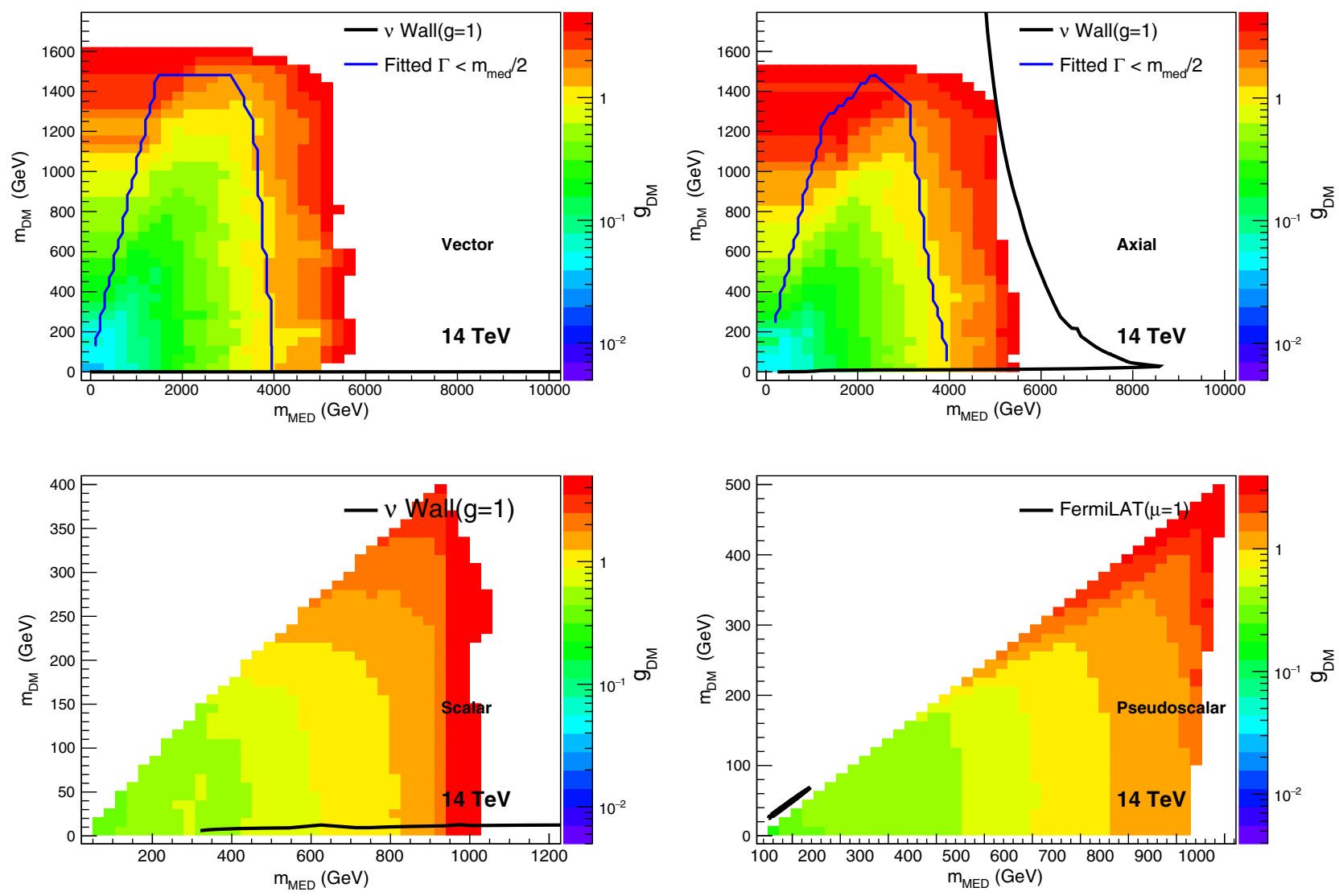

FIG. 7. Exclusion limits on mediator mass versus dark matter mass for given couplings based on multijet measurements at 14 TeV LHC. We show results for vector (upper left panel), axial-vector (upper right panel), scalar (lower left panel) and pseudoscalar (lower right panel) mediators. Plots for vector and axial-vector mediators also show the regions inside which $\Gamma_{\text {MED,min }}<m_{\mathrm{MED}}$ so that the particle physics interpretation of (axial)-vector mediators applies. Scalar and pseudoscalar mediators widths we use are sufficiently narrow.

In Fig. 6 we also distinguish between the monojet (shown in green) and the multijet-based analyses (shown in yellow). It can be seen that the new multijet-based analysis is more powerful and provides a considerable improvement at 14 and at $100 \mathrm{TeV}$. It readily follows from Fig. 6 that the new multileg analysis provides a considerable improvement at $14 \mathrm{TeV}$ relative to the results of our earlier work [4]. At $100 \mathrm{TeV}$ the exclusion limits in Fig. 6 are completely new, and we also point out that the $\mu$-factor remains $\lesssim 1$ well into the $\mathrm{TeV}$ range of mediator masses even for scalar and pseudoscalar mediators. The impact of including the additional matrix elements in the signal simulation is, as predicted, much greater for the FCC, which allows for copious production of light degrees of freedom. For the case of scalar and pseudoscalar mediators at $14 \mathrm{TeV}$ there is a cross-over for mediators heavier than $\simeq 1 \mathrm{TeV}$, which is absent at $100 \mathrm{TeV}$. This corresponds to exactly the regions of phase space in which the off-shell effects dominate. The one-jet sample has access to the significant cross section which arises from the tails of the Breit-Wigner distribution, whereas the multileg sample does not. This region therefore has large theory errors using the multileg sample. However, we note that the region of phase space for which the multileg sample breaks down is far from the values of $\mu=1$, so this region of phase space is of limited importance in regards to setting limits on model parameters. Finally we note that Fig. 6 also includes cross sections for interesting SM predictions which the FCC and Run II of the LHC will investigate. We present the cross sections for $t \bar{t} H$ and $H H$ and show their relative size compared to our DM predictions.

Figure 7 shows the $14 \mathrm{TeV}$ LHC exclusion limits for all four mediator models interpreted on the $\left(m_{\mathrm{MED}}, m_{\mathrm{DM}}\right)$ plane, and the color-coding indicates the value of the mediator coupling, assuming $g_{\mathrm{DM}}=g_{\mathrm{SM}}$. To provide a benchmark for the collider reach for searches of DM, we compare their projected exclusion contours with those relevant to the present and future direct and indirect detection experiments. Specifically, for the cases of scalar, vector and axial-vector mediators we show the neutrino wall which is expected to correspond to the ultimate reach of the direct detection (DD) experiments. For the pseudoscalar mediators one should compare with the indirect detection (ID) (to avoid the nonrelativistic velocity 

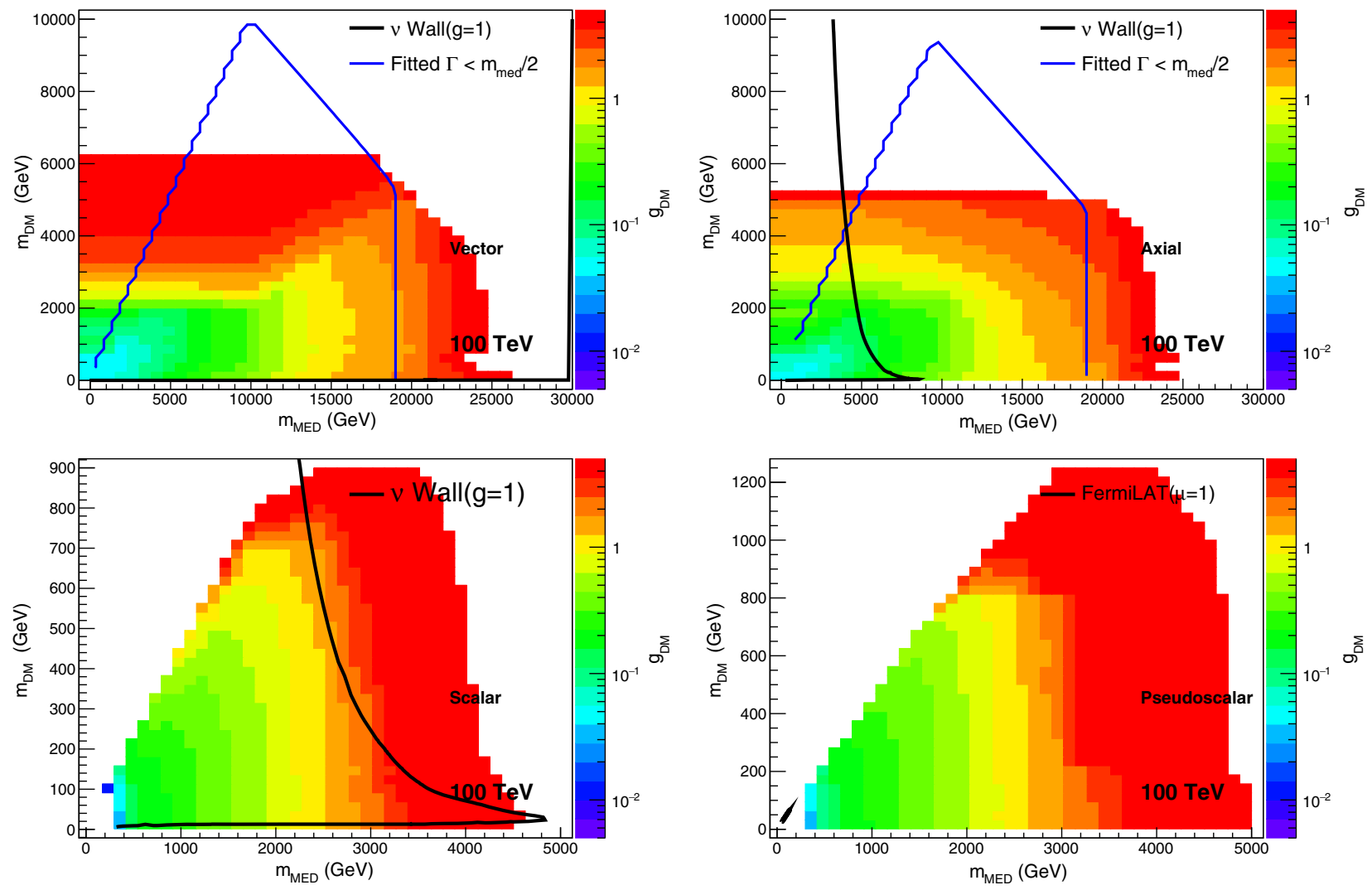

FIG. 8. $100 \mathrm{TeV}$ exclusion limits on mediator mass versus dark matter mass for a given coupling g based on multijet measurements. We show results for vector (upper left panel), axial-vector (upper right panel), scalar (lower left panel) and pseudoscalar (lower right panel). Vector and axial-vector mediators plots indicate the regions where the minimal mediator widths do not exceed $m_{\mathrm{MED}} / 2$, thus reducing the available parameter space reach to $m_{\mathrm{DM}} \lesssim m_{\mathrm{MED}} / 2$ and cutting off large values of the couplings. For (pseudo)scalar mediators the width we use is always sufficiently narrow.

suppression affecting pseudoscalar cross sections in DD experiments). Hence for the pseudoscalar mediators we show the indirect detection limits using FERMI-LAT data [72]. Figure 8 generalizes the results to the FCC case with $100 \mathrm{TeV}$ energy. We find large improvements in reach for the mediator mass $m_{\mathrm{MED}}$ as well as the dark matter mass $m_{\mathrm{DM}}$. Increasing the center-of-mass energy from 14 to $100 \mathrm{TeV}$ allows us to probe 4-5 times larger mediator and dark matter masses, irrespective of the mediator's spin. It is interesting to note that the limits obtained for the neutrino wall for vector, axial and scalar mediators included completely those of the LHC operating at $14 \mathrm{TeV}$. That is to say that, given enough time, data and understanding of astrophysical backgrounds, DD experiments can ultimately outperform the LHC for these mediator types (albeit with several model dependent caveats which render the results complementary to one anther in many regards). On the other hand, for the FCC large regions of parameter space can be accessed for the axial and scalar mediator which cannot be accessed by DD experiments limited by the neutrino wall. This extension of the parameter space for these types of models is a significant benefit of the FCC since it is unlikely this parameter region will ever be reached by current generations of experiments. Finally we note that as the couplings grow larger the width of the mediator grows, eventually for large enough values of the coupling it can exceed $m_{\mathrm{MED}} / 2$ and a particle interpretation of the mediator is no longer valid. We highlight these regions for the vector and axial vector case. For the scalar case, which corresponds to our multileg on-shell sample the limit is trivially imposed by the on-shell condition (for the region where $m_{\mathrm{DM}}>m_{\mathrm{MED}} / 2$ ). We therefore only present the contours for the cases where they are relevant.

In Figs. 9 and 10 we show these exclusion contours for the characteristic to a simplified model analysis fixed value of the mediator couplings, $g_{\mathrm{DM}}=g_{\mathrm{SM}}=1$ for all 4 mediator models of Eqs. (1)-(4). To enable the direct comparison between different experiments/techniques, these figures show all five exclusion contours-the $14 \mathrm{TeV}$ and the $100 \mathrm{TeV}$ limits, using both the one-jet and the multijet analysis, together with the DD/ID noncollider limits/projections.

It is interesting to note the dependence of the DD limits in the scalar mediator case on the number of quark degrees of freedom it couples to. Unlike the production mechanism at collider searches which is sensitive only to the heavy top 

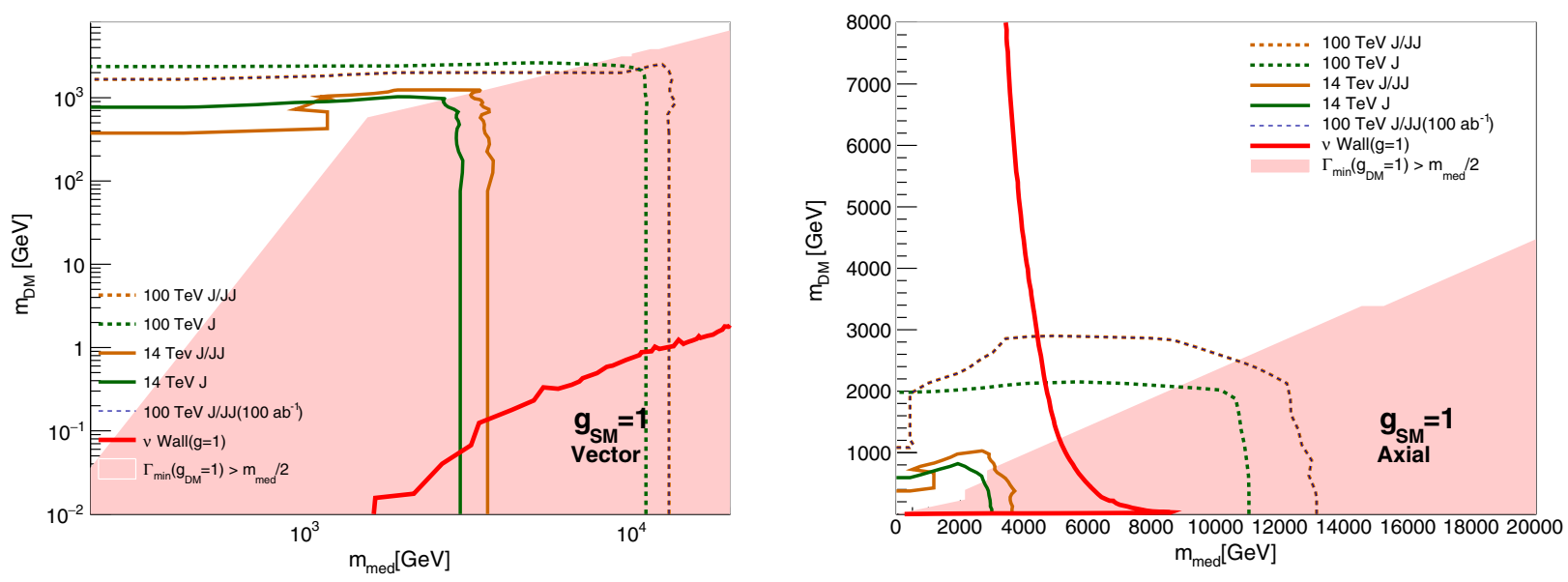

FIG. 9. Mass limits for vector mediator models (left panel) and axial-vector models (right pannel) at 14 and $100 \mathrm{TeV}$ colliders using the multileg and a single-leg analysis. We also show the neutrino wall limit of the direct detection.
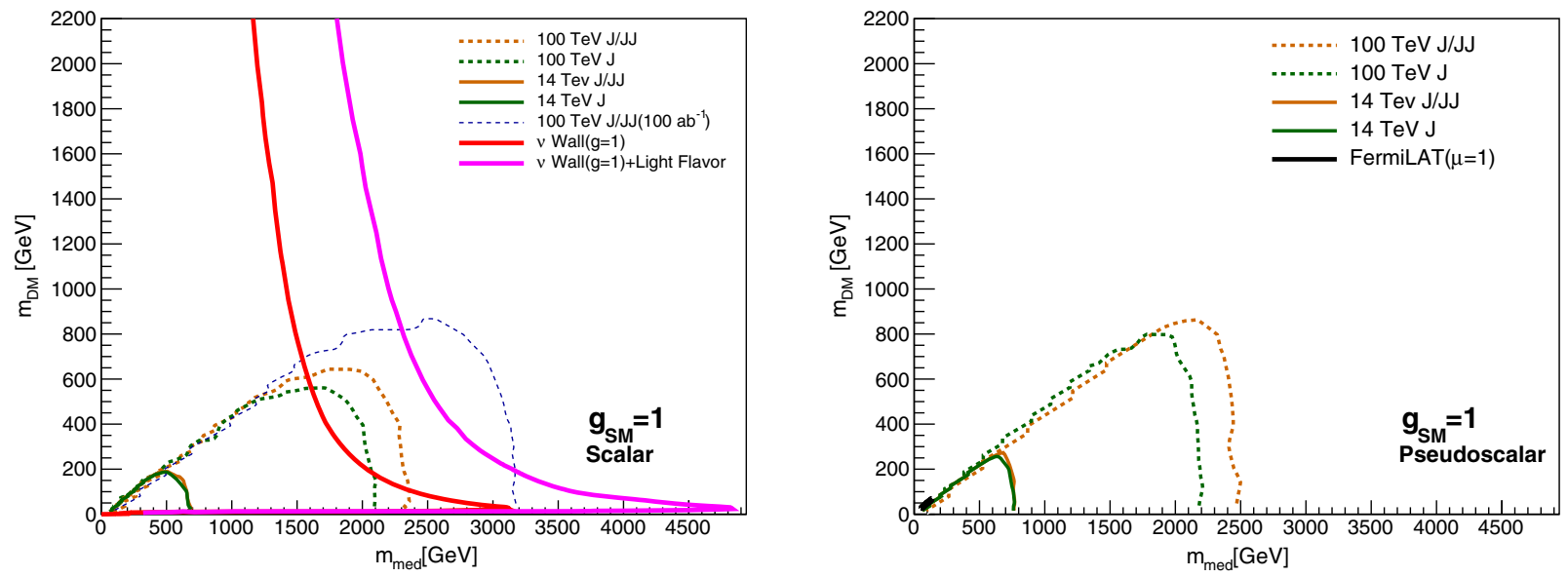

FIG. 10. Mass limits for scalar mediator models (left panel) and pseudoscalar models (right panel) at 14 and $100 \mathrm{TeV}$ colliders using the multileg and a single-leg analysis. The neutrino wall affecting the direct detection experiments is shown in the left plot and the indirect detection limit for pseudoscalars using FERMI-LAT data [72] is shown as a tiny speck in the lower left of the plot on the right.

quark, the DD limits are sensitive also to light degrees of freedom thanks to the cancellation of the quark mass in the $y_{q} / m_{q}$ factor in Eq. (12). Thus, the DD limits are quite sensitive to choice of flavors that mediator couples to in the simplified model. The magenta contour in Figure 9 represents the inclusion of interactions with all quark flavors [as in the simplified model in Eq. (1)]. For a different choice of the simplified model, for example with only the top quark couplings to the mediator, the DD contour is shown in red. The difference between the red and magenta contours in the scalar mediator case in Fig. 9 shows the sensitivity of the DD limits to a range of simplified models; at the same time the collider searches are primarily sensitive to the scalar-totop couplings. ${ }^{5}$ For this parameter choice we note that the collider constraints lie below the neutrino wall for $1 \mathrm{ab}^{-1}$,

\footnotetext{
${ }^{5} \mathrm{We}$ note that in the previous figures the $\nu$-wall curve corresponds to the magenta curve.
}

as the FCC collects more data the wall can be breached. As an example we plot the expected limit given $100 \mathrm{ab}^{-1}$ of FCC data for the scalar mediator.

Finally in Fig. 11 we show the plots in terms of the spindependent and the spin-independent DM-neutron cross sections for a more traditional comparison of collider limits in terms of our simplified models with the limits/projections from the direct and indirect detection experiments. We compare the results in the $\sigma, m_{\mathrm{DM}}$ plane. The general pattern of Figs. 9-10 is reproduced, with the $\nu$-wall for the spin-independent cases providing the strongest projected bounds. For the axial-vector and scalar mediators(with TeV-scale mediator masses as chosen in Fig. 11) our LHC contours cross the neutrino wall limits of direct detection experiments for $m_{\mathrm{DM}} \lesssim 10 \mathrm{GeV}$. At $100 \mathrm{TeV}$ we find that collider bounds for the axial mediator are the strongest and universally below the $\nu$-wall limit of direct detection, whilst for these parameter choices the scalar mediator and the 

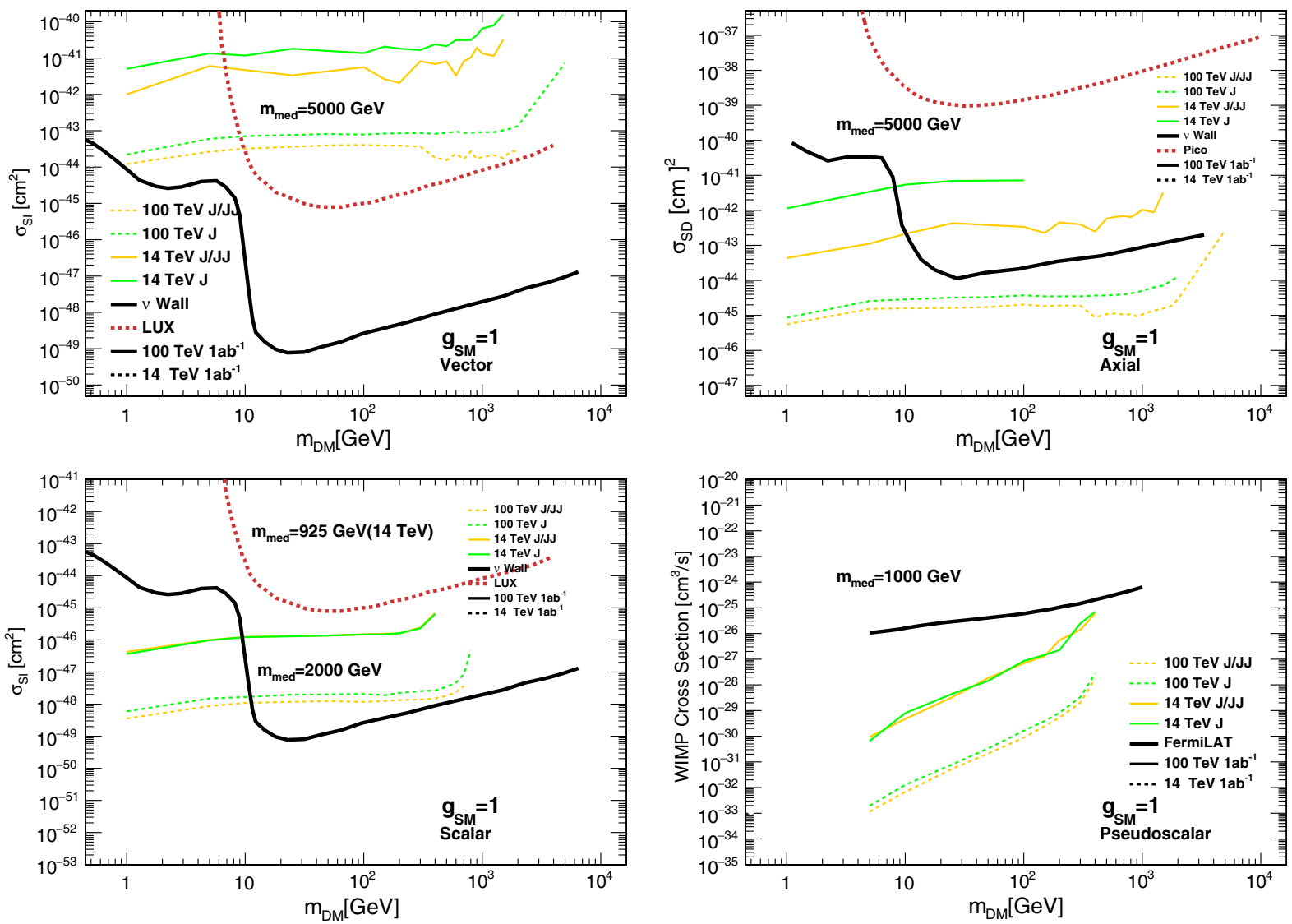

FIG. 11. Collider exclusion contours interpreted in terms of the spin-dependent and spin-independent cross sections plotted as functions of the dark matter mass. For the vector, axial-vector and scalar mediator models indicate the neutrino wall limit of the direct detection experiments. For the pseudoscalar mediator model we show the indirect detection limits (using FERMI-LATdata [72]).

$\nu$-wall are comparable. In the pseudoscalar case the last plot in Fig. 11 demonstrates that both $14 \mathrm{TeV}$ and $100 \mathrm{TeV}$ collider bounds provide a multiorder of magnitude improvement over the current ID reach.

\section{CONCLUSIONS}

We have presented a comprehensive study of the forthcoming and future hadron collider limits and projections at 14 and $100 \mathrm{TeV}$ for searchers of new physics associated with the dark matter sector. The dark sectors are characterised in this work in terms of four generic classes of simplified models Eqs. (1)-(4) where interactions between the standard model partons and the "invisible" dark matter sector particles are described by four basic types of mediators: scalar, pseudoscalar, vector or axial-vector particles. The dark matter particles we consider are produced via $s$-channel mediator exchange, see Fig. 1. For collider searches of dark particles to be effective, two body decays of mediators produced on-shell should be kinematically possible, which requires that $m_{\mathrm{MED}}>2 m_{\mathrm{DM}}$, as can be seen in e.g. Figs. 7. It is then the ability to produce the mediator particle itself that underlines the efficiency of collider searches for dark matter, and not so much the particular species of dark matter the mediator decays into, in so far as these decays are kinematically allowed. Importantly, there is no requirement that once produced, the mediators should decay predominantly into the cosmologically stable dark matter, instead (and arguably more plausibly) they can have significant branching ratios for decay into any dark sector particles which are long-lived on collider scales. Hence we do not impose the relic density constraints on the dark particle production in our simplified model treatment.

Collider limits on the signal cross-sections for the cases of the $14 \mathrm{TeV}$ LHC and the $100 \mathrm{TeV}$ FCC are summarized in Fig. 6 for all four mediator types. From these we have determined the collider reach on the $\left(m_{\mathrm{DM}}, m_{\mathrm{MED}}\right)$ mass plane at $14 \mathrm{TeV}$ in Fig. 7 and $100 \mathrm{TeV}$ in Fig. 8. Both these sets of figures compare the collider reach with the neutrino wall limit of direct detection experiments and the current data from the indirect detection (in the latter case for the pseudoscalar mediator models). We conclude that for scalar and axial-vector mediators collider searches are highly competitive and also complimentary to direct detection experiments, while in the pseudoscalar case the collider limits are unchallenged. These figures also show the required strength of the DM coupling of the mediators, 
which in all cases remains largely in the perturbative regime.

Figures 9 and 10 combine the $14 \mathrm{TeV}$ and $100 \mathrm{TeV}$ projections for collider searches and compare them with DD and ID limits at fixed values of mediator couplings $g_{\mathrm{DM}}=g_{\mathrm{SM}}=1$. For vector and axial-vector mediators mediator masses of up to $\sim 3 \mathrm{TeV}$ can be probed at the LHC and extended to nearly $13 \mathrm{TeV}$ at the $100 \mathrm{TeV}$ FCC. For scalar and pseudoscalar mediators, the $14 \mathrm{TeV}$ LHC reach is of $m_{\mathrm{MED}} \sim 700 \mathrm{GeV}$ and up to $\sim 4-5 \mathrm{TeV}$ for the FCC. Thus the future hadron circular collider would be able to truly probe the few- $\mathrm{TeV}$ scales of the dark matter sectors, and in this regime it crosses over and goes beyond the projected limits of DD and ID experiments as can be seen from Figs. 9-10 on the $m_{\mathrm{DM}}, M_{\mathrm{MED}}$ mass plane and the plots in Fig. 11 which interpret collider searches in the language of DD experiments in terms of the spinindependent and spin-dependent cross-sections. Our FCC limits were presented assuming a rather modest data set of $1 \mathrm{ab}^{-1}$, over the lifetime of the machine a much larger data set should be collected, and the limits/discovery sensitivity will be significantly enhanced.
In summary, although our results for the FCC are somewhat speculative, it is clear that there is a huge potential for such a machine to probe a large parameter space for a variety of dark sector scenarios. Indeed an FCC style machine can provide access to regions of parameter space for axial and scalar mediators which lie beyond the potential limits of the cosmic neutrino wall. Such a possibility is an exciting aspect of the emerging physics program of the FCC, and in our opinion represents a strong motivation to press ahead with its ultimate construction.

\section{ACKNOWLEDGMENTS}

We would like to thank Oliver Buchmueller, Krisitan Hahn, Michelangelo Mangano, Christopher McCabe, Filip Moortgaart, Nick Wardle, and Nhan Tran, for valuable discussions. The research of V. V. K. and M. S. is supported by STFC through the IPPP grant and for V. V. K. by the Wolfson Foundation and Royal Society. V. V. K. acknowledges the Aspen Center for Physics supported by National Science Foundation Grant No. PHY-1066293, where this work was finalized.
[1] P. A. R. Ade et al. (Planck Collaboration), Planck 2015 results. XIII. Cosmological parameters, arXiv:1502.01589.

[2] G. Bertone, D. Hooper, and J. Silk, Particle dark matter: Evidence, candidates and constraints, Phys. Rep. 405, 279 (2005).

[3] M. Benedikt, in the FCC week 2015, Washington D.C., 2329 March 2015, http://indico.cern.ch/event/340703/session/ 108/contribution/186 D. Schulte, in the FCC week 2015, Washington D.C., 23-29 March 2015 http://indico.cern.ch/ event/340703/session/109/contribution/189 CEPC/SppC preliminary conceptual design report, http://cepc.ihep.ac.cn/ preCDR/volume.html.

[4] P. Harris, V. V. Khoze, M. Spannowsky, and C. Williams, Constraining dark sectors at colliders: Beyond the effective theory approach, Phys. Rev. D 91, 055009 (2015).

[5] M. Low and L. T. Wang, Neutralino dark matter at $14 \mathrm{TeV}$ and $100 \mathrm{TeV}$, J. High Energy Phys. 08 (2014) 161.

[6] J. Bramante, P. J. Fox, A. Martin, B. Ostdiek, T. Plehn, T. Schell, and M. Takeuchi, Relic neutralino surface at a 100 TeV collider, Phys. Rev. D 91, 054015 (2015).

[7] Q. F. Xiang, X. J. Bi, P. F. Yin, and Z. H. Yu, Searches for dark matter signals in simplified models at future hadron colliders, Phys. Rev. D 91, 095020 (2015).

[8] A. Freitas, S. Westhoff, and J. Zupan, Integrating in the Higgs portal to fermion dark matter, J. High Energy Phys. 09 (2015) 015.

[9] M. Cirelli, F. Sala, and M. Taoso, Wino-like minimal dark matter and future colliders, J. High Energy Phys. 10 (2014) 033; 01 (2015) 041(E).
[10] J. Abdallah et al., Simplified models for dark matter and missing energy searches at the LHC, arXiv:1409.2893.

[11] S. Malik et al., Interplay and characterization of dark matter searches at colliders and in direct detection experiments, Phys. Dark Univ. 9-10, 51 (2015).

[12] O. Buchmueller, M. J. Dolan, S. A. Malik, and C. McCabe, Characterising dark matter searches at colliders and direct detection experiments: Vector mediators, J. High Energy Phys. 01 (2015) 037.

[13] M. R. Buckley, D. Feld, and D. Goncalves, Scalar simplified models for dark matter, Phys. Rev. D 91, 015017 (2015).

[14] U. Haisch and E. Re, Simplified dark matter top-quark interactions at the LHC, J. High Energy Phys. 06 (2015) 078 .

[15] M. Chala, F. Kahlhoefer, M. McCullough, G. Nardini, and K. Schmidt-Hoberg, Constraining dark sectors with monojets and dijets, J. High Energy Phys. 07 (2015) 089.

[16] V. V. Khoze, G. Ro, and M. Spannowsky, Spectroscopy of scalar mediators to dark matter at the LHC and at $100 \mathrm{TeV}$, Phys. Rev. D 92, 075006 (2015).

[17] O. Buchmueller, S. A. Malik, C. McCabe, and B. Penning, Constraining the Fermi-LAT Excess with Multi-jet Plus MET Collider Searches, Phys. Rev. Lett. 115, 181802 (2015).

[18] J. Fan, S. M. Koushiappas, and G. Landsberg, Pseudoscalar portal dark matter and new signatures of vector-like fermions, J. High Energy Phys. 01 (2016) 111.

[19] O. Lebedev and Y. Mambrini, Axial dark matter: The case for an invisible Z?, Phys. Lett. B 734, 350 (2014). 
[20] J. Abdallah et al. (DM@LHC Collaboration), Simplified models for dark matter searches at the LHC, Phys. Dark Univ. 9-10, 8 (2015).

[21] D. Abercrombie et al. (ATLAS/CMS Dark Matter Forum), Dark matter benchmark models for early LHC Run-2 searches: Report of the ATLAS/CMS dark matter forum, arXiv:1507.00966.

[22] G. D’Ambrosio, G. F. Giudice, G. Isidori, and A. Strumia, Minimal flavor violation: An effective field theory approach, Nucl. Phys. B645, 155 (2002).

[23] V. M. Abazov et al. (D0 Collaboration), Search for Large Extra Dimensions in the Monojet + Missing $E_{T}$ Channel at D0, Phys. Rev. Lett. 90, 251802 (2003).

[24] T. Aaltonen et al. (CDF Collaboration), A Search for Dark Matter in Events with One Jet and Missing Transverse Energy in $p \bar{p}$ Collisions at $\sqrt{s}=1.96 \mathrm{TeV}$, Phys. Rev. Lett. 108, 211804 (2012).

[25] S. Chatrchyan et al. (CMS Collaboration), Search for dark matter and large extra dimensions in monojet events in $p p$ collisions at $\sqrt{s}=7 \mathrm{TeV}$, J. High Energy Phys. 09 (2012) 094.

[26] G. Aad et al. (ATLAS Collaboration), Report No. ATLASCONF-2012-084.

[27] V. Khachatryan et al. (CMS Collaboration), Search for dark matter, extra dimensions, and unparticles in monojet events in proton-proton collisions at $\sqrt{s}=8 \mathrm{TeV}$, Eur. Phys. J. C 75, 235 (2015).

[28] E. Diehl (ATLAS Collaboration), The search for dark matter using monojets and monophotons with the ATLAS detector, AIP Conf. Proc. 1604, 324 (2014).

[29] J. L. Feng, S. Su, and F. Takayama, Lower Limit on Dark Matter Production at the Large Hadron Collider, Phys. Rev. Lett. 96, 151802 (2006).

[30] Q. H. Cao, C. R. Chen, C. S. Li, and H. Zhang, Effective dark matter model: Relic density, CDMS II, Fermi LAT and LHC, J. High Energy Phys. 08 (2011) 018.

[31] M. Beltran, D. Hooper, E. W. Kolb, Z. A. C. Krusberg, and T. M. P. Tait, Maverick dark matter at colliders, J. High Energy Phys. 09 (2010) 037.

[32] J. Goodman, M. Ibe, A. Rajaraman, W. Shepherd, T. M. P. Tait, and H. B. Yu, Constraints on light Majorana dark matter from colliders, Phys. Lett. B 695, 185 (2011).

[33] J. Goodman, M. Ibe, A. Rajaraman, W. Shepherd, T. M. P. Tait, and H.B. Yu, Constraints on dark matter from colliders, Phys. Rev. D 82, 116010 (2010).

[34] P. J. Fox, R. Harnik, J. Kopp, and Y. Tsai, Missing energy signatures of dark matter at the LHC, Phys. Rev. D 85, 056011 (2012).

[35] U. Haisch, F. Kahlhoefer, and J. Unwin, The impact of heavy-quark loops on LHC dark matter searches, J. High Energy Phys. 07 (2013) 125.

[36] G. C. Branco, P. M. Ferreira, L. Lavoura, M. N. Rebelo, M. Sher, and J. P. Silva, Theory and phenomenology of two-Higgs-doublet models, Phys. Rep. 516, 1 (2012).

[37] V. Silveira and A. Zee, Scalar phantoms, Phys. Lett. 161B, 136 (1985).

[38] R. Schabinger and J. D. Wells, A minimal spontaneously broken hidden sector and its impact on Higgs boson physics at the Large Hadron Collider, Phys. Rev. D 72, 093007 (2005).
[39] B. Patt and F. Wilczek, Higgs-field portal into hidden sectors, arXiv:hep-ph/0605188.

[40] C. Englert, T. Plehn, D. Zerwas, and P. M. Zerwas, Exploring the Higgs portal, Phys. Lett. B 703, 298 (2011).

[41] C. Englert, J. Jaeckel, V. V. Khoze, and M. Spannowsky, Emergence of the electroweak scale through the Higgs portal, J. High Energy Phys. 04 (2013) 060.

[42] T. Hambye and A. Strumia, Dynamical generation of the weak and dark matter scale, Phys. Rev. D 88, 055022 (2013).

[43] C. D. Carone and R. Ramos, Classical scale-invariance, the electroweak scale and vector dark matter, Phys. Rev. D 88, 055020 (2013).

[44] V. V. Khoze, C. McCabe, and G. Ro, Higgs vacuum stability from the dark matter portal, J. High Energy Phys. 08 (2014) 026.

[45] M. Carena, A. Daleo, B. A. Dobrescu, and T. M. P. Tait, $Z^{\prime}$ gauge bosons at the Tevatron, Phys. Rev. D 70, 093009 (2004).

[46] A. Avetisyan et al., Methods and results for standard model event generation at $\sqrt{s}=14 \mathrm{TeV}, 33 \mathrm{TeV}$ and $100 \mathrm{TeV}$ proton colliders (A Snowmass Whitepaper), arXiv:1308.1636.

[47] J. Alwall, R. Frederix, S. Frixione, V. Hirschi, F. Maltoni, O. Mattelaer, H.-S. Shao, T. Stelzer, P. Torrielli, and M. Zaro, The automated computation of tree-level and next-toleading order differential cross sections, and their matching to parton shower simulations, J. High Energy Phys. 07 (2014) 079.

[48] P. J. Fox and C. Williams, Next-to-leading order predictions for dark matter production at hadron colliders, Phys. Rev. D 87, 054030 (2013).

[49] J. M. Campbell, R. K. Ellis, and C. Williams, MCFM website http://mcfm.fnal.gov.

[50] J. Baglio et al., Release Note - VBFNLO 2.7.0, arXiv: 1404.3940; J. Baglio et al., VBFNLO: A parton level Monte Carlo for processes with electroweak bosons-Manual for Version 2.7.0, arXiv:1107.4038; VBFNLO: A parton level Monte Carlo for processes with electroweak bosons, Comput. Phys. Commun. 180, 1661 (2009).

[51] T. Sjostrand, S. Mrenna, and P.Z. Skands, A brief introduction to PYTHIA 8.1, Comput. Phys. Commun. 178, 852 (2008).

[52] R. D. Ball et al. (NNPDF Collaboration), Parton distributions for the LHC Run II, J. High Energy Phys. 04 (2015) 040.

[53] M. Backovic, M. Krämer, F. Maltoni, A. Martini, K. Mawatari, and M. Pellen, Higher-order QCD predictions for dark matter production at the LHC in simplified models with s-channel mediators, Eur. Phys. J. C 75, 482 (2015).

[54] O. Mattelaer and E. Vryonidou, Dark matter production through loop-induced processes at the LHC: the s-channel mediator case, Eur. Phys. J. C 75, 436 (2015).

[55] J. Butler, D. Contardo, M. Klute, J. Mans, and L. Silvestris, Reports No. CERN-LHCC-2015-010, No. LHCC-P-008.

[56] G. Aad et al. (ATLAS Collaboration), Reports No. CERNLHCC-2012-022, No. LHCC-I-023.

[57] V. Khachatryan et al. (CMS Collaboration), Performance of the CMS missing transverse momentum reconstruction in pp data at $\sqrt{s}=8 \mathrm{TeV}$, J. Instrum. 10, P02006 (2015).

[58] V. Khachatryan et al. (CMS Collaboration), Determination of Jet Energy Calibration and Transverse Momentum Resolution in CMS, J. Instrum. 6, 11002 (2011). 
[59] V. Khachatryan et al. (CMS Collaboration), Report No. CMS-PAS-EXO-12-055.

[60] V. Khachatryan et al. (CMS Collaboration), Report No. CMS-PAS-EXO-14-004.

[61] V. Khachatryan et al. (CMS Collaboration), Report No. CMS-PAS-SUS-12-002.

[62] A.L. Read, Presentation of search results: The CL(s) technique, J. Phys. G 28, 2693 (2002).

[63] G. Cowan, K. Cranmer, E. Gross, and O. Vitells, Asymptotic formulae for likelihood-based tests of new physics, Eur. Phys. J. C 71, 1554 (2011); 73, 2501(E) (2013).

[64] H. Y. Cheng and C.W. Chiang, Revisiting scalar and pseudoscalar couplings with nucleons, J. High Energy Phys. 07 (2012) 009.

[65] O. Buchmueller, M. J. Dolan, and C. McCabe, Beyond effective field theory for dark matter searches at the LHC, J. High Energy Phys. 01 (2014) 025.

[66] A. Kurylov and M. Kamionkowski, Generalized analysis of weakly interacting massive particle searches, Phys. Rev. D 69, 063503 (2004).

[67] J. Hisano, K. Ishiwata, and N. Nagata, Gluon contribution to the dark matter direct detection, Phys. Rev. D 82, 115007 (2010).

[68] K. Cheung, C. T. Lu, P. Y. Tseng, and T. C. Yuan, Collider constraints on the dark matter interpretation of the CDMS II results, arXiv:1308.0067.
[69] A. Crivellin, M. Hoferichter, and M. Procura, Accurate evaluation of hadronic uncertainties in spin-independent WIMP-nucleon scattering: Disentangling two- and threeflavor effects, Phys. Rev. D 89, 054021 (2014).

[70] M. Hoferichter, J. Ruiz de Elvira, B. Kubis, and U. G. Meiner, High-precision Determination of the Pion-nucleon $\sigma$-term from Roy-Steiner Equations, Phys. Rev. Lett. 115, 092301 (2015).

[71] P. Cushman et al., Snowmass CF1 summary: WIMP dark matter direct detection, arXiv:1310.8327.

[72] M. Ackermann et al. (Fermi-LAT Collaboration), Constraining Dark Matter Models from a Combined Analysis of Milky Way Satellites with the Fermi Large Area Telescope, Phys. Rev. Lett. 107, 241302 (2011).

[73] A. A. Abdo et al. (Fermi-LAT Collaboration), Observations of Milky Way Dwarf Spheroidal galaxies with the FermiLAT detector and constraints on dark matter models, Astrophys. J. 712, 147 (2010).

[74] J. M. Zheng, Z. H. Yu, J. W. Shao, X. J. Bi, Z. Li, and H. H. Zhang, Constraining the interaction strength between dark matter and visible matter: I. fermionic dark matter, Nucl. Phys. B854, 350 (2012).

[75] C. Boehm, M. J. Dolan, C. McCabe, M. Spannowsky, and C. J. Wallace, Extended gamma-ray emission from Coy dark matter, J. Cosmol. Astropart. Phys. 05 (2014) 009. 